Maria E. Xagorari-Gleißner,

Institut für Klassische Archaeologie der

Universität Erlangen-Nürnberg, Germany

UDK 904:726.8"652"

m.xagorari@gmx.net

73.032.044(38)"-04/-03"

Wissenschaftliche Originalarbeit

Abgegeben: August 22, 2010

Akzeptiert: November 02, 2010

\title{
DIE ERFORSCHUNG DER ATTISCHEN GRABKUNST KLASSISCHER ZEIT: EIN ÜBERBLICK
}

\begin{abstract}
Mehr als 3500 attischen Grabmäler klassischer Zeit (430-317 v. Chr.) sind uns heute erhalten: Reliefierte Grabvasen, naiskosförmige Reliefs, reliefierte Bildfeldstelen und weitere einfachen Reliefs vermitteln einen Eindruck der athenischen Gesellschaft in der Zeit der Demokratie. Seit den Anfängen der griechischen Archäologie ziehen sie selbstverständlich das Interesse der traditionellen altertumswissenschaftlichen Forschung. Im folgenden Aufsatz werden die heutigen Forschungstendenzen und Problemen skizziert.
\end{abstract}

SChlüSSElWÖRTER: Griechenland; KLASSIK; ATtische GrabKunST.

\section{FORSCHUNGSGESCHICHTE DER ATTISCHEN GRABKUNST}

Die attischen Grabmäler, speziell die Reliefs (Abb. 1), sind schon seit dem späten 19. Jahrhundert Thema wissenschaftlicher Abhandlungen. Sie sind in Corpora ${ }^{1}$ und Datenbanken (Arachne) gesammelt, die ständig mit neu entdecktem Material bereichert werden. Dass diese Monumente in Attika hergestellt wurden, ist außer Zweifel, da ihr Material attisch ist, nämlich pentelischer oder hymettischer Marmor oder anderer lokaler Stein. Durch Inschriften ist gesichert, dass ein großer Teil der Monumente Athener Bürgern und ein kleiner Teil

1 Conze 1893, CAT 1993, ARMA 2009, SEMA 2006.
Nicht-Athenern - Fremden, Sklaven, Metöken ${ }^{2}$ - gehörte (Abb. 2). Die Namensinschriften auf attischen Grabmäler sind von statistisch untersucht worden ${ }^{3}$. Die Forscherin kam zum Ergebnis, dass Namensinschriften im späten 5. Jh. noch spärlich waren, aber seit den 60er Jahren des 4. Jhs. zunahmen. In dieser Steigerung sieht die Autorin das wachsende politische Bewusstsein der Athener, die durch ihren Namen auf ihren Grabmälern bekannt werden wollten. Die Gründe für dieses Verhalten sind allerdings nicht geklärt. $\mathrm{Zu}$ betonen ist, dass mehr als die Hälfte der Inschriften weder Demotikon noch Ethnikon tragen, so dass der genaue Status der Besitzer

2 Fragiadakis 1988.

3 Meyer 1993. 
vieler Namenstelen unbekannt bleibt.

In den zahlreichen bisherigen Abhandlungen über die attischen Grabmäler ${ }^{4}$ werden erwartungsgemäß die attischen Bild- und Formelemente hervorgehoben und die gesamte Gattung als Zeugnis der Selbstpräsentation der Athener Bürger betrachtet ${ }^{5}$. Der attische Einfluss auf lykische, karische und sidonische Grabmäler $^{6}$ sowie auf die neuzeitliche Grabkunst ${ }^{7}$ ist schon in der Literatur notiert ${ }^{8}$. Ob aber einzelne Bildelemente, wie z. B. Waffen, Geräte und Gefäße, als einzige Dekorelemente oder ganz individuelle Szenen auch von anderen Kulturkreisen beeinflusst wurden, ist bisher wenig untersucht worden ${ }^{9}$ (Abb. 3). Allgemein ist das Ausmaß des Einflusses anderer Völker auf das Griechenland klassischer Zeit noch nicht vollständig untersucht worden.

\section{DIE GRIECHEN UND IHRE NACHBARN}

Der Hellenozentrismus prägte die Forschung des 19. und 20. Jahrhunderts. Der ist einigermaßen legitim, zumal die Mehrheit der antiken schriftlichen Quellen von griechischen Autoren und in der griechischen Sprache überliefert sind, die Auskunft über die auswärtigen Tätigkeiten der Griechen berichten. Durch Apoikies (= Kolonien) und Emporia (= Handelsniederlassungen) sorgten die Griechen für die Verbreitung ihrer Produkte und ihrer Kultur.

4 Grundlegend: Diepolder 1931, Schmaltz 1970, Stupperich 1977, Schmaltz 1983, Kokula 1984, Scholl 1996, Bergemann 1997, Bäbler 1998, Himmelmann 1999, Adak 2003, Posamentir 2006.

5 Besonders Bergemann 1997.

$6 \mathrm{Maaß} 2008$.

7 Hildebrandt 2007.

8 Dazu auch die Arbeiten über den sog. Klagefrauensarkophag von Sidon mit seinen attischen Stilelementen: Fleischer 1983.

9 Vgl. Melonenfrisur als importiertes Bildelement in die attische Kunst aus der Region der Skythen oder der Thraker: Lopes 2009.

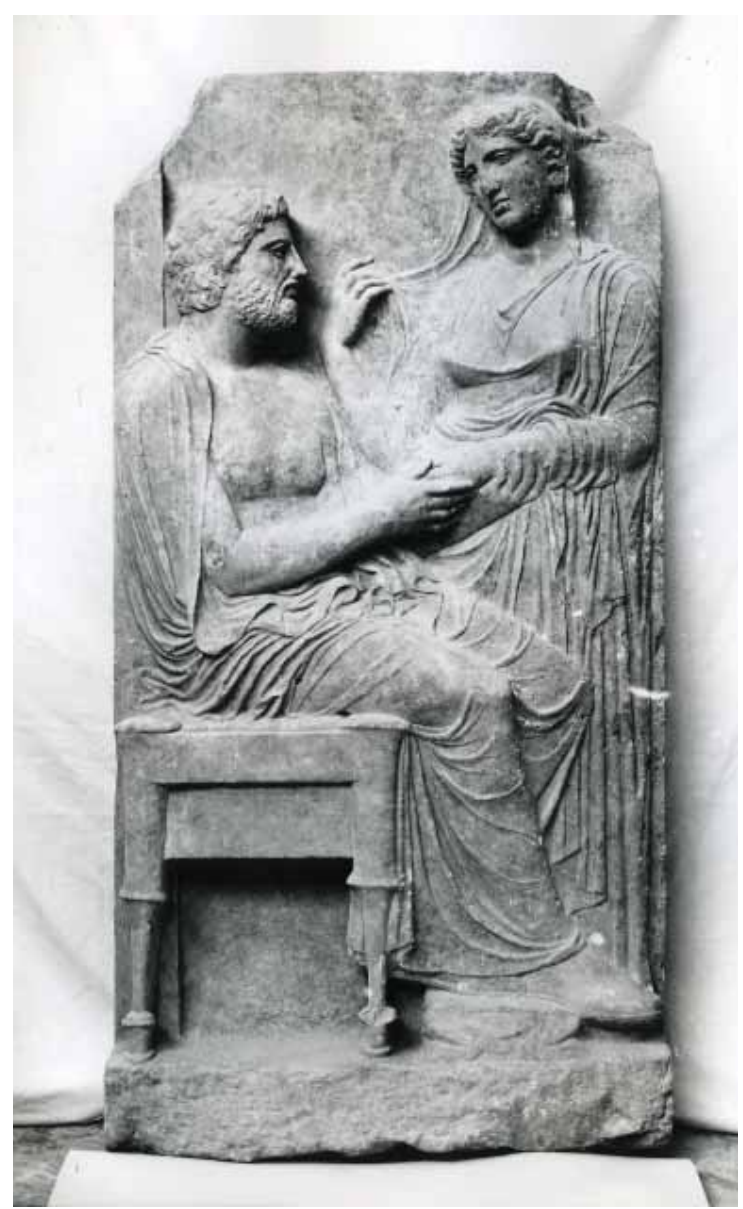

Abb. 1. Attisches Grabrelief eines Ehepaares, frühes 4. Jh. v. Chr., Athen Nationalmuseum, Inv. 4507.

Der Export griechischer Produkte, vor allem von Wein und Olivenöl sowie von Luxusgütern wie Grabstelen, ist an vielen Orten der antiken Welt belegt ${ }^{10}$. Die letzten Jahren aber interessiert sich die Forschung für die Interaktion der Griechen mit ihren Nachbarn in den Kolonien und den Handelsniederlassungen ${ }^{11}$. Darüber hinaus wird die Kultur von nicht-griechischen Völkern und Stämmen intensiv untersucht ${ }^{12}$ und in der Öffentlichkeit präsentiert ${ }^{13}$. Da die Makedonen

10 Vgl. Boardman 1999, Petropoulos 2005, Grammenos and Petropoulos 2003, Pogiatzi 2003, Carter and Posamentir 2006, Kreuz 2007.

11 Vgl. Tsetskhladze 2006, Ders. 2008, Bilde and Petersen 2008, Morin 2009.

12 Perser: Miller 1997, Bakir 2001. Thraker: Bouzek 2005, Popov and Fol 2010. Lykien: Seyer 2009.

13 Vgl. Ausstellungen über die Phönizier: Gehrig and Nie- 


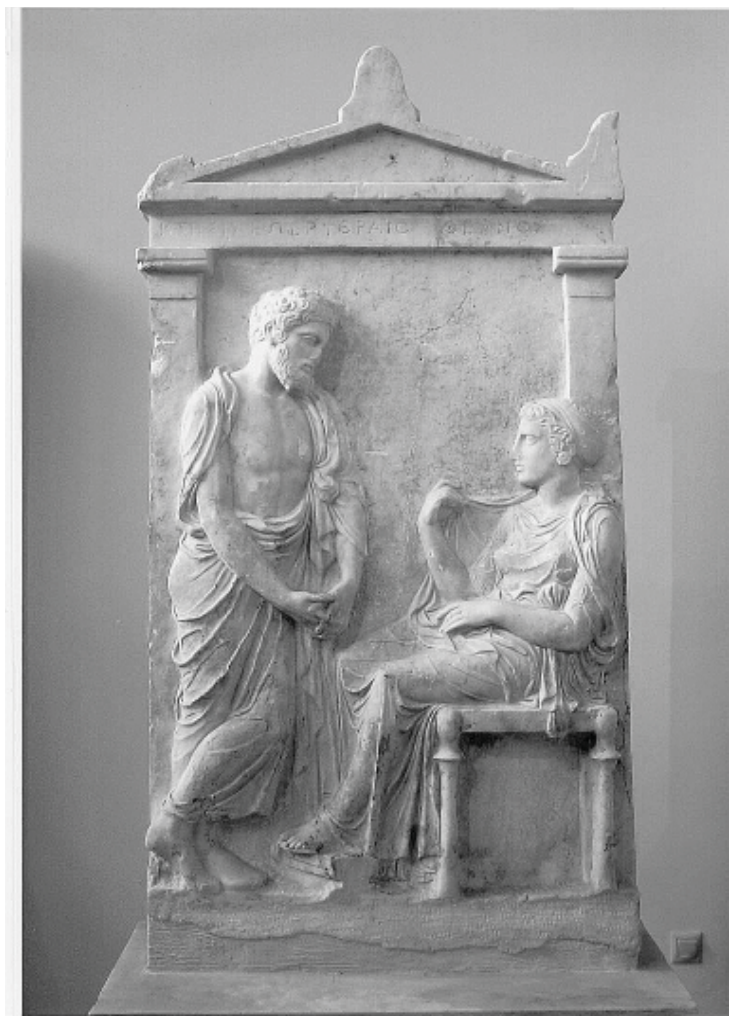

Abb. 2. Attische Grabreliefstele des Ktesileos aus Erythrae (Böotien) und der Theano, um $400 \mathrm{v}$. Chr., Athen Nationalmuseum, Inv. 3472.

eine aufkommende Macht bildeten, wird der Einfluss der makedonischen Kunst unter anderen auch auf die attische Kunst und Lebensweise notiert und manchmal sogar überbetont ${ }^{14}$.

Die Erforschung der Kultur der nicht-griechischen Völker warf eine neue Forschungsfrage auf, nämlich die des ethnischen Selbstbewusstseins der Menschen in der Antike $^{15}$. Dabei scheint aber eine Definition des Begriffs „ethnicity“ noch unklar - oder sogar oft unpassend - zu sein. Der Begriff ist z. B. für die Kolonisten geeignet ${ }^{16}$, denn diese Gruppe setzt eine gemeinsame geographische Herkunft oder einen gemeinsamen Vorfahren voraus. Mit

meier 1990, die Skythen: Danailov 2004, die Perser: Koch 2006.

14 Palagia and Tracy 2003.

15 Hahn 1993, Hall 1997, Fless and Treister 2005, Halles and Hodos 2010.

16 Gosden 2004. dem Bewusstsein einer ethnischen Identität als Ausgangspunkt kommt ANTONACCIO zum Ergebnis, dass eine andere Menschengruppe, die Oberschicht, eine „transcultural hybridity“ bildet, da sie eher durch ihren Sinn nach Luxus, habrosyne, gekennzeichnet wird ${ }^{17}$. Die Diskussion über die Identität in der Antike geht also weiter.

\section{DIE GRABNAISKOI: FORSCHUNG MIT PERSPEKTIVE}

Eine neue Form von Grabmälern taucht kurz vor der Mitte des 4. Jhs. v. Chr. auf, die Naiskoi (Abb. 4). Eine kurze Auflistung des Materials legte DESPINES in einem kurzen Aufsatz im Neugriechischen $\operatorname{vor}^{18}$. Darin geht der Forscher auf keine Analyse des Materials ein, bietet aber für viele seiner aufgelisteten Stücke eine gute photographische Dokumentation. Der Autor erwähnt eine Reihe von vollplastischen fragmentiert erhaltenen Figuren, die er aus technischen Gründen - nämlich wegen ihrer eher flachen und grob bearbeiteten Rückseite insgesamt einunddreißig Naiskoi zuordnet. Dem Forscher geht es nicht um eine Rekonstruktion von Naiskoi, sondern allein um die Erkenntnis, dass diese Gattung von Grabmälern im spätklassischen Attika ziemlich verbreitet war. Seine Liste ist nicht vollständig; die Stücke aus dem Kerameikos, wie z. B. der Naiskos der Philoumene aus dem Grabbezirk der Messenier ${ }^{19}$ sind nicht eingeschlossen. Ausnahme, die die Regel bestätigt, ist der Naiskos des sog. Persers ${ }^{20}$. Despines datiert alle diese Grabmäler aus stilistischen Gründen in das letzte Drittel des 4. Jhs. v. Chr. (also 330-300 v. Chr.). Diese Datierung ist zwar interessant, aber problematisch. Interessant, weil dadurch manche Naiskoi in die lykurgische Zeit (338-325 v. Chr.) und manche in die Zeit der makedonischen

\footnotetext{
17 Antonaccio 2010.

18 Despines 2002.

19 Kovacsovics 1990.

20 Scholl 2000.
} 
Herrschaft über Athen (ab 322 v. Chr.) hineinfallen. Problematisch, weil die politische Situation in Athen in diesen Jahren sehr stark schwang, nämlich von der unabhängigen Polis in der Zeit Lykurgs zum Anhänger einer globalisierten Welt unter den Makedonen. Der Athener Staatsmann Lykurg hatte in den Jahren 338-325 v. Chr. die politische Führung inne und leistete enormen Widerstand gegen die makedonische Herrschaft. Sein Widerstand lag weniger im militärischen, sondern mehr im politischen Bereich. Dabei versuchte er, das Bewusstsein der Athener und der in Attika wohnhaften Fremden - der Metöken - zu stärken und das positive Image Athens in der Außenwelt wiederherzustellen. Sein Programm ist in der attischen öffentlichen Baukunst ersichtlich und sowohl bei antiken Schriftstellern überliefert als auch in der neuzeitlichen Literatur erkannt. In einer Gesellschaft, in der der Bürger sich durch seine öffentliche Funktion verstand, ist zu fragen, wie die Politik Lykurgs auf die private Grabkunst der Bürger, privilegierten Fremden und der NichtBürger einwirkte. Darüber hinaus durften nach dem Grabluxusgesetz des Demetrius Phalereus (317-307 v. Chr.) monumentale Grabmäler nicht mehr errichtet werden. Demetrius Phalereus wurde von den Makedonen in seine Position als Statthalter eingesetzt. Wenn er also die luxuriösen und monumentalen Grabmäler verboten hat, sind die monumentalen Naiskoi nicht in das letzte Drittel des 4. Jhs. sondern nur in die lykurgische Zeit zu datieren. Und dies ist ein erklärungsbedürftiges Phänomen.

Die Datierung der attischen Grabmäler generell ist ein Problem bei ihrer Erforschung, da sie auf der Basis einer zweifelhaften stilistischen Entwicklung basiert. Die Mehrheit dieser Monumente wurden im späten 19. Jh. gefunden und einfach gesammelt. Ein Versuch, diese Werke in einen Fundkontext einzuordnen, wurde damals nicht gemacht, da die Forschung andere kunsthistorische - Akzente setzte. In den Corpora von Conze wird oft eine allgemeine Fundangabe

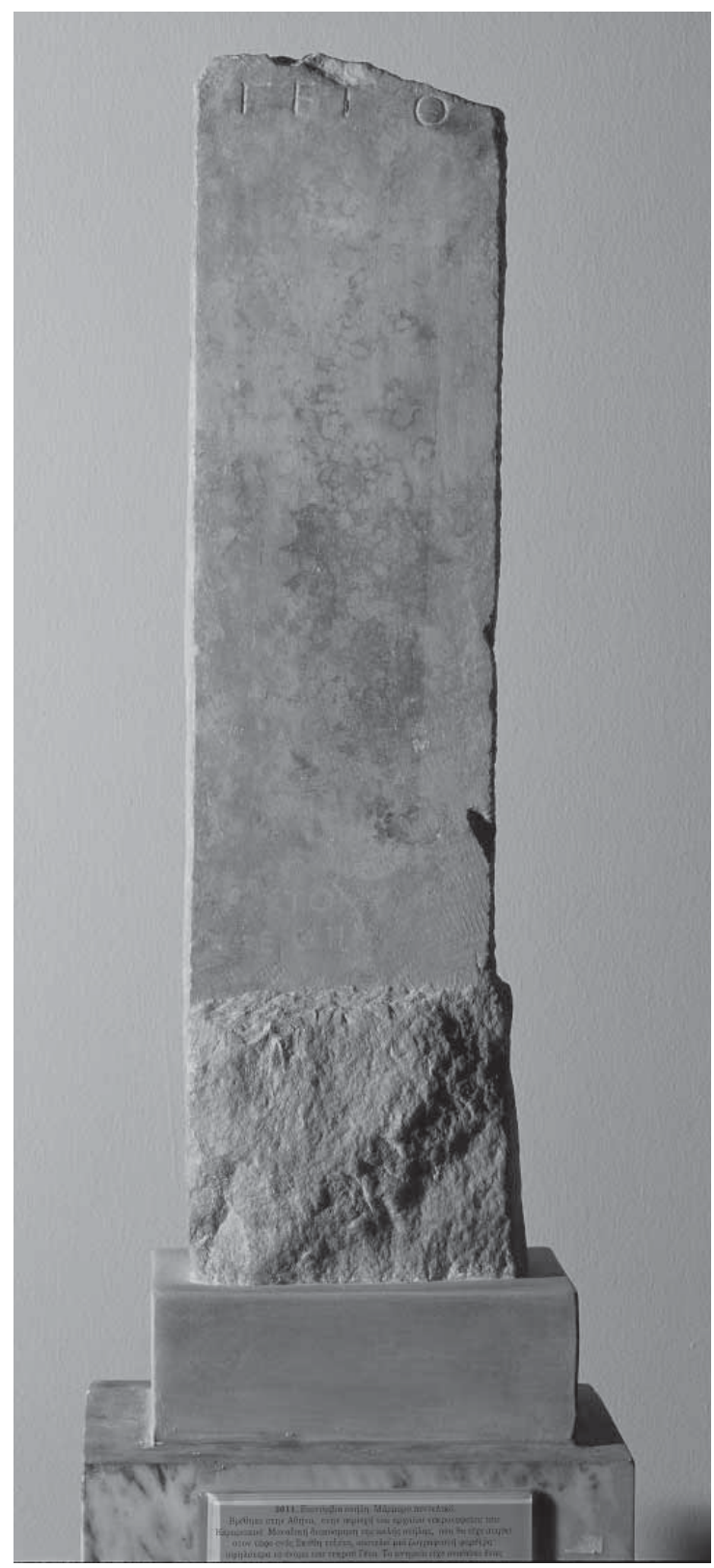

Abb. 3. Attische bemalte Grabstele eines Geten beschriftet "Geto", um 400 v. Chr., Athen Nationalmuseum, Inv. 2611.

gemacht, z. B. Athen; Kerameikos; Nordfriedhof vom Piräus und ähnliches. Der Fundkontext wurde nicht berücksichtigt. Einen Versuch einer näheren Bestimmung der Fundorte haben Petrakos und seine Mitarbeiter neulich gemacht ${ }^{21}$, allerdings ohne Erfolg bei den meisten Monumenten. Die zeitliche Anordnung jener Grabkunstgattung

21 SEMA 2006. 


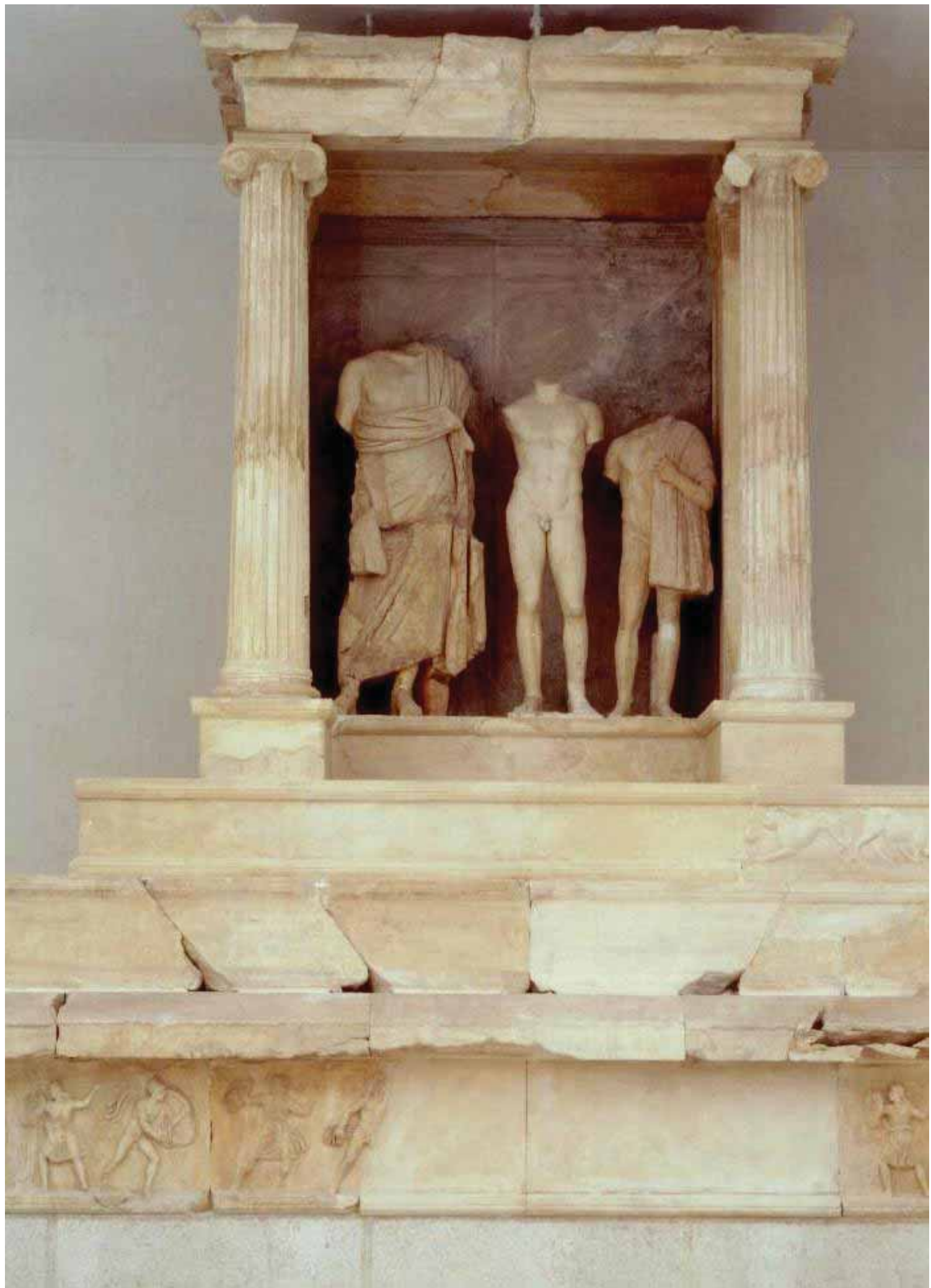

Abb. 4. Attischer Grabnaiskos des Nikeratos aus Istros und seines Sohnes Polyxenos, um 330-317 v. Chr., Piräus Museum Inv. 2448+2447+2451. 
sowie die Frage nach der Identität ihrer Besitzer gehören zu den Aufgaben der Forschung in der Zukunft.

\section{ZUSAMMENFASSUNG}

Das Feld der archäologischen Forschung ist heute sehr weit und breit und tauchen immer viele neuen Fragen auch für die alten Funden auf. Das gilt vor allem für die attischen Grabmäler klassischer Zeit, die trotz ihrer hundertjährigen Erforschung immer wieder von Neuem entdeckt werden.

\section{LITERATURVERZEICHNIS}

Adak, M. 2003

Metöken als Wohltäter Athens. Untersuchungen zum sozialen Austausch zwischen ortsansässigen Fremden und der Bürgergemeinde in klassischer und hellenistischer Zeit, ca. 500-150 v. Chr., München: Tuduv

\section{Antonaccio, C. M. 2010}

(Re)Defining Ethnicity: Culture, Material Culture, and Identity, S. Hales and T. Hodos, (ed.), Material Culture and social Identities in the Ancient World, Cambridge: Cambridge University Press, 32-53

\section{ARMA 2007}

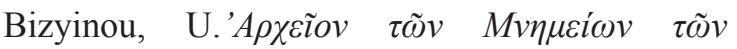

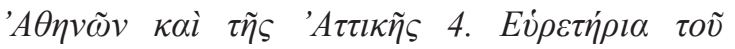

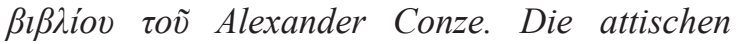
Grabreliefs (Berlin 1893-1922), Athen: En Athinais Archaeologike Etaireia

Bäbler, B. 1998

Fleissige Thrakerinnen und wehrhafte Skythen. Nichtgriechen im klassischen Athen und ihre archäologische Hinterlassenschaft, StuttgartLeipzig: Teubner
Bakir, T. and Sancisi-Weerdenburg, H. and Gürtekin, G. and Briant, P. and Henkelman, W. 2001

(ed.), Achaemenid Anatolia, Proceedings of the First International Symposium on Anatolia in the Achaemenid Period, Bandırma 15 - 18 August 1997. Leiden : Nederlands Instituut voor het Nabije Oosten

\section{Bergemann, J. 1997}

Demos und Thanatos. Untersuchungen zum Wertsystem der Polis im Spiegel der attischen Grabreliefs des 4. Jahrhunderts v. Chr. und zur Funktion der gleichzeitigen Grabbauten, München: Brinkmann and Biering

Bilde, G. P. and Petersen, J. H. 2008

Meetings of Cultures in the Black Sea Region. Between conflicts and Coexistence, Black Sea Studies 8, Aarus: Aarhus University Press

\section{Boardman, J. 1999 ${ }^{4}$}

The Greeks Overseas. Their Early Colonies and Trady ( $4^{\text {th }}$ ed.), London: Thames and Hudson

\section{Bouzek, J. 2005}

Thracians and their Neighbours: Their Destiny, Art and Heritage, StHercyn 9, Prague: Selbstverlag

\section{Carter, J. C. and Posamentir, R. 2006}

The Gravestones of Chersonesos. Research and Conservation, Istanbul: Ege Yayınları

\section{CAT 1993}

Clairmont, C. W., Classical Attic Tombstones, Kilchberg: Akanthus

\section{Conze, A. 1893-1922}

Die attischen Grabreliefs, Berlin: Spemann

\section{Danailov, B. 2004}

Die Thraker. Das goldene Reich des Orpheus, Ausstellung 23. Juli - 28. November 2004 
Kunst- und Ausstellungshalle der Bundesrepublik Deutschland in Bonn, Bonn: Kunst und Ausstellungshalle der BRD

\section{Despines, G. 2002}

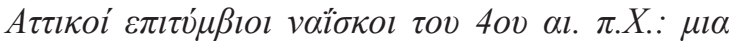

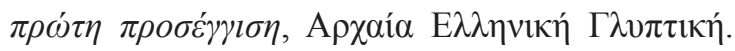
A

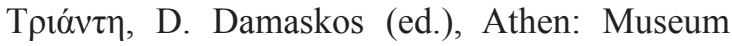
Benaki, 209-231

\section{Diepolder, H. 1931}

Die attischen Grabreliefs des 5. und 4. Press

Jahrhunderts v. Chr., Berlin:

\section{Fleischer, R. 1983}

Der Klagefrauensarkophag aus Sidon, IstForsch 34, Tübingen: Wasmuth

Fless, F. - Treister, M. (ed.) 2005

Bilder und Objekte als Träger kultureller Identität und interkultureller Kommunikation im Schwarzmeergebiet. Kolloquium in Zschortau/ Sachsen 13.2.-15.2.2003, Rahden: Leidorf

Fragiadakis, C. 1988

Die attischen Sklavennamen von der spätarchaischen Epoche bis in die römische Kaiserzeit. Eine historische und soziologische Untersuchung, Athen: Selbstverlag

\section{Gehrig, U. - Niemeier, H. G. 1990}

Die Phönizier im Zeitalter Homers, Ausstellungskatalog Kestner-Museum, Hannover 14. Sept.-25. Nov. 1990, Mainz a. R: von Zabern

\section{Gosden, C. 2004}

Archaeology and Colonialism: Cultural Contact from $5000 \mathrm{BC}$ to the Present, Cambridge: Cambridge University Press

Grammenos, D. V. - Petropoulos, E. K. 2003 Ancient Greek Colonies in the Black Sea,
Thessaloniki: Archaeological Institute of Northern Greece

Hahn, I. 1993

Ethnische Identität, Integration und Dissimilation im Lichte der Namengebung, Annales Universitatis scientiarum Budapestinensis. Sectio historica 26, 9-17

Hales, S. - Hodos, T. (ed.) 2010

Material Culture and social Identities in the Ancient World, Cambridge: Cambridge University

\section{Hall, J. M. 1997}

Ethnic Identity in Greek Antiquity, Cambridge: Cambridge University Press

\section{Hildebrandt, F. 2007}

Klassizistischer Baustil in Griechenland und die attischen Namenstelen, Thetis 13/14, Ruhpolding: Rutzen, 176-185

\section{Himmelmann, N. 1999}

Attische Grabreliefs, Opladen: Westdeutscher Verlag

\section{Koch, A. (ed.) 2006}

Beigleitheft zur Ausstellung „Pracht und Prunk der Großkönige. Das persische Weltreich", Stuttgart: Historisches Museum der Pfalz Speyer

\section{Kokula, G. 1984}

Marmorlutrophoren, 10. Beih. AM, Berlin: Gebr. Mann

\section{Kovacsovics, W. K. et al. 1990}

Die Eckterrasse an der Gräberstrasse des Kerameikos, Kerameikos. Ergebnisse der Ausgrabungen 14, Berlin: de Gruyter 


\section{Kreuz, P. A. 2007}

Klassische Grabstelen im Bosporanischen Reich: Grabmarkierung und Repräsentation am Grab, Griechen. Skythen. Amazonen, Ausstellung 14. Juni - 21. Oktober 2007 im Pergamonmuseum Berlin, U. Kästner - M. Langner - B. Rabe (ed.), Berlin: Freie Universität

\section{Lopes, E. 2009}

Ethinicity Reconsidered: the Melon Coiffure from the Hyperboreans to Central Greece, Numismatica e Antichità Classiche, Quaderni Ticinesi 38, Selbstverlag 27- 48

\section{Maaß, M. 2008}

Der Orient und die Bildhauerkunst der griechischen Klassik, Die Geschichte der antiken Bildhauerkunst II, P. C. Bol (ed.), Mainz a. R.: von Zabern 475-493

Meyer, E. A. 1993

Epithaphs and citizenship in classical Athens, JHS 113, London: Society for the Promotion of Hellenic Studies, 99 - 121

Miller, M. C. 1997

Athens and Persia in the fifth Century B. C. A Study in Cultural Receptivity, Cambridge: Cambridge University Press

\section{Morin, J. 2009}

The Greek Expansion to the Black Sea, AJA 113, New York: Archaeological Institute of America, 129-133

\section{Palagia, O. - Tracy, S. V. (ed.) 2003}

The Macedonians in Athens 322-229 B.C. Proceedings of an international Conference held at the University of Athens, 24.-26. Mai 2001, Oxford: Oxbow
Petropoulos, E. K. 2005

Hellenic Colonization in Euxeinos Pontos: Penetration, Early Establishment and the Problem of the „Emporion" revisited, Oxford: Archaeopress

\section{Pogiatzi, E. 2003}

Die Grabreliefs auf Zypern von der archaischen bis zur römischen Zeit, Peleus 23, MöhnseeWamel: Bibliopolis

\section{Popov, D. - Fol, V. 2010}

The Deities of the Thracians, Sofia: TANGRA Publ. House

\section{Posamentir, R. 2006}

Bemalte attische Grabstelen klassischer Zeit, München: Brinkmann und Biering

Schmaltz, B. 1970

Untersuchungenzudenattischen Marmorlekythen, Berlin: Gebr. Mann

\section{Schmaltz, B. 1983}

Griechische Grabreliefs, Darmstadt: Wissenschaftliche Buchgesellschaft

\section{Scholl, A. 1996}

Die attischen Bildfeldstelen des 4. Jhs. v.Chr. Untersuchungen $z u$ den kleinformatigen Grabreliefs im spätklassischen Athen, 17. Beih. AA, Berlin: Gebr. Mann

\section{Scholl, A. 2000}

Der "Perser" und die ,skythischen Bogenschützen " aus dem Kerameikos, JdI 115, Berlin: de Gruyter, 79-112

\section{SEMA 2006}

Bardani, B. N. -Papadopulos, G. K. -Petrakos, W. Ch. (ed.), $\Sigma v \mu \pi \lambda \eta \dot{\rho} \omega \mu \alpha \tau \tilde{\omega} v \dot{\varepsilon} \pi \imath \tau \nu \mu \beta i \omega v \mu \nu \eta \mu \varepsilon i \omega v$ $\tau \tilde{\eta} \varsigma$ A $\tau \tau \iota \kappa \tilde{\eta} \varsigma$, Athen: En Athenais Archaeologike Etaireia 
Seyer, M. 2009

Zur Ausstattung der Kammern lykischer

Felsgräber, IstMitt 59, Tübingen: Wasmuth

Stupperich, R. 1977

Staatsbegräbnis und Privatgrabmal im

klassischen Athen, Münster: Selbstverlag

Tsetskhladze, G. R. 2006:

Greek Colonisation. An account of Greek Colonies and other Settlements Overseas Bd. 1, Leiden: Brill

Tsetskhladze, G. R. 2008

Greek Colonisation. An account of Greek Colonies and other Settlements Overseas Bd. 2, Leiden: Brill

\section{REZIME}

ISTRAŽIVANJE ATIČKE

NADGROBNE UMETNOSTI KLASIČNOG PERIODA: PREGLED

KlJuČne reči: AtiKa, nadgrobna umetnost, KLASIČAN PERIOD, METECI

Do danas je sačuvano preko 3500 nadgrobnih spomenika klasičnog perioda (430. do 317. g.p.n.e.): reljefne nadgrobne vaze, reljefi u obliku naiskosa, reljefne stele sa prikazima izdeljenim u polja i ostali jednostavni reljefi prenose sliku atičkog društva iz perioda demokratije. Od početka grčke arheologije, oni naravno privlače interesovanje tradicionalnog proučavanja klasike. U ovom tekstu će biti skicirane današnje tendencije u istraživanju i problemi vezani za njih. 